\title{
用多徏强红外激光离解 $\mathrm{CHCIF}_{2}$ 分离 ${ }^{13} \mathrm{C}$ 同位素
}

\author{
杨立书迋正民 刘宗才 吴传秀 \\ (中国科学院安微光学棈密机械研究所,合肥)
}

\section{关垏词分离 ${ }^{\mathrm{B}} \mathrm{C}$ 同位素、多频红外激光、分步共振激发}

在数光分离 ${ }^{33} \mathrm{C}$ 同位素中, 寻求改善多光子离解选择性的新途径同时增加其效率是当前人 们最关心的问题之一. 作者在文献 [1] 中首次报道用简单的办法在单台 $\mathrm{TEACO}$ ，激光器上 同时输出 $9 P(22), 9 P(20), 9 P(18), 9 P(16), 9 P(14)$ 支线, 并用它们辐 照 $\mathrm{CF}_{3} \mathrm{I}$, 在 室温 下所得 ${ }^{13} \mathrm{C}$ 的浓缩系数是单频结果的 14.5 倍. Letokhov 等 ${ }^{[2]}$ 用间隔开的多支线（如 $9 R(8)$, $9 R(20)$ 和 $9 P(34)$ 或 $9 R(8), 9 R(16), 9 R(24)$ 和 $9 P(34)$ 等)激发 $\mathrm{CHClF}$ ，分子也大大 地提高了离解选择性和产率, 这种方法虽然具有较大的优越性. 但是, 在实验中由于用了丽台 TEACO，激光器, 使实猃装置复杂化. 多频激光与分子相互作用之所以能大大地提高选择性, 是由于能用多个激发频率分步地与分子的最低的几个分离振动能级共振或近共振激发, 从而 克服了在单频激光场中激发频率与振动能级失谐的困难. 多频技术不仅能改善多光子离解选 择性，而且也是制备很高激发态分子的有力手段.9.1.

鉴于 $\mathrm{CHClF}_{2}$ 体系可以在较高气压下进行同位素分离, 没有复杂的副反应, 原料廉价, 来源方便等优点, 在实际的工业规模分离 ${ }^{13} \mathrm{C}$ 同位素中, 它可能是一种较为理想的介质. 另 外, 它的红外吸收谱在 TEACO 激光 $9 R(20)$ 支线附近有一宽而强的吸收带. 我们选用 $\mathrm{TEACO}_{2}$ 激光 $9 R(24), 9 R(22), 9 R(20), 9 R(18), 9 R(16)$ 五支线同时辐照 $\mathrm{CHClF}_{2}$ 分

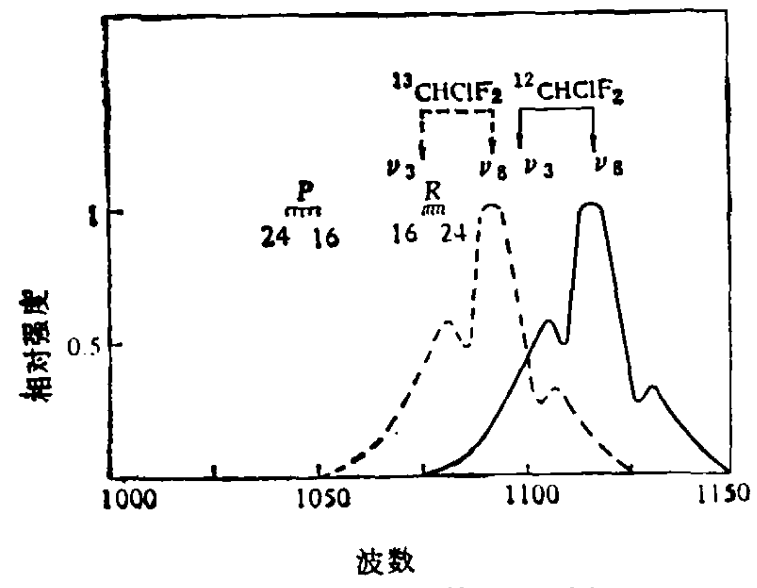

图 $1 \mathrm{CHClF}_{2}$ 分子的红外吸收谱

子, 共振激发 ${ }^{13} \mathrm{CHClF}_{2}$, 在室温下, ${ }^{13} \mathrm{C}$ 的洨 缩系数达到 230 倍, 且相应的产率为 $40 \%$, 它们与单频的结果相比有数量级的提高. 我 们还提出了影响选择性的机制主要是 “转动 瓶颈”效应。

\section{一、实 验}

工业用瓶装 $\mathrm{CHClF}_{2}$ 分子经冷凉, 抽真 空的多次循环操作,除去杂质后用于实验。它 的红外吸收谱 ${ }^{[2]}$ 示于图 1. ${ }^{12} \mathrm{CHClF}$, 分子的 $\nu_{8}=1115 \mathrm{~cm}^{-1}, \nu_{3}=1100 \mathrm{~cm}^{-1}$. 由于同位 素的光谱位移， ${ }^{3} \mathrm{CHClF}_{2}$ 的 $\nu_{3} \approx 1076 \mathrm{~cm}^{-1}$, 并且是一宽而强的吸收带, 它与 $\mathrm{TEACO}_{2}$ 激 本文1987 年 8 月 27 日收到. 
光 $9.4 \mu$ 带 $R$ 支的几乐强线波长都匹配，所以在实验中我们用 $\mathrm{TEACO}$ 激光 $9 R(24) ， 9 R \times$ (22)，9R(20)，9R(18)，9R(16) 五支线组成的多频激光辐照 $\mathrm{CHClF}_{2}$ 分子,辐照后的样品 由质谱和红外光谱分析. 为了比较, 我们也角 $9 P(24), 9 P(22) ， 9 P(20) ， 9 P(18), 9 P(16)$ 五支线非共振激发 ${ }^{13} \mathrm{CHClF}_{2}$ 分子。实验装置和 $\mathrm{TEACO}_{2}$ 激光器性能详见文献[1].

\section{二、结果和讨论}

多频强红外场离解 $\mathrm{CHClF}_{2}$ 分子的机理是:

$$
\begin{aligned}
& \mathrm{CHCl}_{2} \mathrm{~F}_{2}+\sum_{i=1}^{3} n_{i} h v_{i} \longrightarrow \mathrm{CF}_{2}+\mathrm{HCl}, \\
& \mathrm{CF}_{2}+\mathrm{CF}_{2} \longrightarrow \mathrm{C}_{2} \mathrm{~F}_{4},
\end{aligned}
$$

(1) 式中 $\nu_{i}$ 表示辐照 $\mathrm{CHClF}_{2}$ 时所用的 $\mathrm{TEACO}_{2}$ 激光的五个频率, 由于它们是从单台激光 器输出, 其同步性能极佳. 由于 $\mathrm{HCl}$ 是稳定产物, 所以, 这一离解过程既简单又无复杂的 副反应,这是该过程具有高效率的因素之一。离解过程的一个很重要的特征量是离解产率, 它 随辐炤脉冲数的增加而增大. 但是, 在实际过程中不能无限制的辐照下去, 在有高产率的情况 下,应尽量减少辐照脉冲数。因此, 要求增大 ${ }^{13} \mathrm{CHClF}_{2}$ 的离解分数. 用多频强红外场分步接 力式地激发分子有可能达到此目的. 因每步的吸收截面是 ${ }^{[3]}$ :

$$
\sigma_{i}\left(\omega_{l i}, \omega_{m i}\right)=\frac{4 \pi^{2} \omega_{L i} d_{i}^{2}}{\hbar c} \frac{\Gamma}{2 \pi} \frac{1}{\left(\omega_{L i}-\omega_{m i}\right)^{2}+\frac{1}{4} \Gamma^{2}},
$$

而每步的激发速率则为

$$
R_{i}=\sigma_{i}\left(\omega_{L i}, \omega_{m i}\right) \cdot F_{i},
$$

(3) 和 (4) 式中 $i=1,2,3,4,5, \omega_{L i}=2 \pi v_{i}$ 是五个激光频率, $\omega_{m i}$ 是分子的五个跃迁 频 率, $d_{i}$ 为跃迁偶极矩阵元, $F_{i}$ 为激光能流密度. 如果在一次脉冲中要求有更多的 ${ }^{13} \mathrm{CHClF}_{2}$ 分 子被激发到离解闻值以上能级,则应尽量增大每步的激发速率 $R_{i}$ 的值. 因此, 应选择恰当的 $\sigma_{i}$ 和 $F_{i}$ 。我们利用 $9 R(24) ， 9 R(22) ， 9 R(20) ， 9 R(18), 9 R(16)$ 五频场离解 ${ }^{13} \mathrm{CHClF}_{2}$ 可 能已达到了此目的.

(2) 式中 ${ }^{12} \mathrm{CF}_{2}$ 和 ${ }^{13} \mathrm{CF}_{2}$ 自由基的不同组合形成 ${ }^{12} \mathrm{C}^{12} \mathrm{CF}_{4},{ }^{12} \mathrm{C}^{13} \mathrm{CF}_{4},{ }^{13} \mathrm{C}^{13} \mathrm{CF}_{4}$ 三种分子, 它们进人四极质谱仪离化后可产生 ${ }^{12} \mathrm{C}^{12} \mathrm{CF}_{3}^{+},{ }^{12} \mathrm{C}^{13} \mathrm{CF}_{3}^{+},{ }^{13} \mathrm{C}^{13} \mathrm{CF}_{3}^{+}$离子, 并分别在荷质比为 $81,82,83$ 处出现很强的质谱峰. 测出各峰的相对值后由公式 [1]

$$
\beta=\frac{1-x}{2 x} \cdot \frac{{ }^{12} \mathrm{C}^{13} \mathrm{CF}_{5}^{+}}{{ }^{12} \mathrm{C}^{12} \mathrm{CF}_{3}^{+}},
$$

可以求出 ${ }^{13} \mathrm{C}$ 同位素的浓缩系数,(5)式中 $x$ 表示 ${ }^{13} \mathrm{CHClF}_{2}$ 分子的天然丰度. 在相同激光能 流密度、不同频率、压力和激光脉冲数条件下所得结果列于表 1 .

从表 1 可以看出,共振激发的浓缩系数和产率都大于非共振激发的结果. 这可由 (3) 和 (4)式得出：共振激发的吸收截面 $\sigma_{i}$ 和激发速率 $R_{i}$ 都大于非共振时的值, 即是说, 每步的 $R_{i}$ 值，共振激发比非共振激发都来得大,最后导致 $R_{5}$ 更大,能使更多的 ${ }^{13} \mathrm{CHClF}_{2}$ 分子被激发到 高能级. 由于高位能级的密度大, 容易使得各个激发频率与相应的能级共振,在准连续区则更 是如此. 对于这种分步共振激发，原则上讲， ${ }^{13} \mathrm{CHClF}_{2}$ 分子都能达到离解间值以上的能级， 以至离解。而对于 ${ }^{12} \mathrm{CHClF}_{2}$ 分子则几乎不被激发, 故被激发的 ${ }^{13} \mathrm{CHClF}_{2}$ 分子的离解选择 
表 1

\begin{tabular}{|c|c|c|c|c|c|}
\hline 实的席昜 & 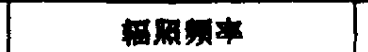 & 气压 & 光脉冲数 & ${ }^{13} \mathrm{C}$ 的波缩系数 & ${ }^{13} \mathrm{C}$ 的产事 \\
\hline 1 & $\mid \begin{array}{c}9 R(24), 9 R(22), 9 R(20) \\
9 R(18), 9 R(16)\end{array}$ & 5.5 & 300 & 230 & $40 \%$ \\
\hline 2 & 同上 & 3.5 & 100 & 208 & $32 \%$ \\
\hline 3 & 同上 & 50 & 300 & 150 & $35 \%$ \\
\hline 4 & 9R(20) & 4 & 300 & 17 & $4.5 \%$ \\
\hline 5 & $\begin{array}{l}9 P(24), 9 P(22), 9 P(20) \\
9 P(18), 9 P(16)\end{array}$ & 6 & 300 & 120 & $20 \%$ \\
\hline 6 & 同上 & 4 & 100 & 90 & $10 \%$ \\
\hline 7 & 同上 & 52 & 300 & 80 & $15 \%$ \\
\hline 8 & $9 P(20)$ & 3.5 & 300 & 6 & $2 \%$ \\
\hline
\end{tabular}

珄和产将会大大地提高。但是,在实际的过程中,由于存在碰撞，两种分子间的 $V-V$ 能量

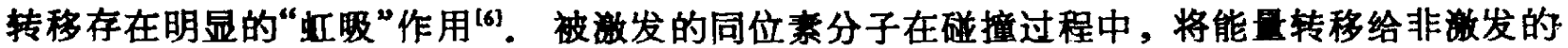
同位素分子后经转动驰豫又重新回到被激光做发而倒空了的能态. 这就使得某一振动能级以 下的一些能级上始终停留了不少分子. 换句话说,在一定程度上不能倒空低能级上的粒子数。 所以，在实际的过程中被敏发的同位素分子由于存在上述“转动瓶颈”效应，使其自身的选择 性和产率的提高受到一定的限制.

\section{* 考文 视}

[1] 刘宗才等,科学通报, 30(1985), 4:261.

[2] Evseev, A. V., Letokhov, V. S. and Puretzky, A. A., Appl. Phyr., B36(1985), 2:93.

[3] Apatin, M. et al., Laser Chem., 5(1985). 231.

[4]杨立书等,科学通报, 31(1986), 8:620.

[5] Ackerhalt, J. R. and Eberly, J. H., Phys. Rev., A14(1976), 5: 1705.

[6]玚立书等,物理学根,36(1987),2:259。 